\title{
Selling Better Bones - the Driving Messages for a Vitamin D3 Supplement
}

\section{Daniel Moskowitz and Howard Moskowitz*}

Szent István University, Budapest, Hungary

*Corresponding Author: Szent István University, Budapest, Hungary.

Received: July 12, 2019; Published: July 16, 2019

DOI: $10.31080 /$ ASPS.2019.03.0342

\begin{abstract}
The paper investigates what types of messages attract consumers, in terms of labelling a vitamin D3 supplement, in terms of presenting its benefits, and in terms of pricing. The paper shows how Mind Genomics uncovers two major segments or mind-sets in the population, and how the messages for one group may not be appropriate for the other group. Finally, the paper introduces cognitive economics, the interaction of pricing, an economic concept, with consumer minds-set regarding the specific product, the vitamin D3 supplement.
\end{abstract}

Keywords: Vitamin D3; Paper; Male; Female

\section{Introduction}

Vitamin D3 is a superstar, with $54 \mathrm{~mm}$ hits on Google (July 3 , 2018). Vitamin D3 has been associated with a variety of good things for the body. Pamela Egan describes the benefits in a simple, albeit commercial pitch for the vitamin:

With all the medical advances in the 20 the century, Vitamin D3 deficiency is still an epidemic. Fifteen to twenty minutes of sunshine each day, in the nude, helps your body manufacture about 10,000 to 15,000 iu's of vitamin D3 per day. The problem is that most people avoid the sun these days due to fears of skin cancer. Just think about plants and what happens to them when they avoid the sun. They wither \& die.

Did you know that vitamin D3 deficiency can result in Obesity, Type 2 Diabetes, High Blood Pressure, Depression, Psoriasis, Fibromyalgia, Chronic Fatigue Syndrome, Kidney Stones, Osteoporosis, \& Neuro-degenerative disease including Alzheimer's Disease. Eventually, Vitamin D deficiency may even lead to Cancer (especially breast, prostate, and colon cancers). Vitamin D3 is believed to play a role in controlling the immune system (possibly reducing one's risk of cancer and autoimmune diseases), increasing neuro-muscular function and decreasing falls, improving mood, protecting the brain against toxic chemicals, and potentially reducing pain.

Vitamin D3 is both a vitamin and a hormone. It acts as a vitamin when it binds with calcium for proper absorption. Humans cannot digest calcium without adequate amounts of Vitamin D3.

The most common reasons for Vitamin D3 deficiency in the United States relates to lack of exposure to sunlight and infrequent consumption of cold water fish such as wild salmon, mackerel and sardines
Source: http://www.pamelaegan.com/articles/vitamin-d3.htm

The foregoing introduction summarizes a great deal of what consumers know about this highly promoted vitamin. The academic medical literature is far richer in detail as can be seen by the literally hundreds of refereed papers dealing with one or another aspect of the effect of Vitamin D3. Here follow references to just a smattering of these papers, most of which have topical interest to the consumer, the person who buys the product in a drugstore, but do not talk about how to sell the vitamin D3 product [1-5].

This paper focuses on the human side of the product, on how people react to messages about Vitamin D, the messages themselves generated in a way that follows what marketers do, not what laboratory scientists do. As such, the paper straddles the fence between the science of the product itself, what the vitamin does, and the science of the mind, namely what is important to people.

\section{Selling vitamin D3}

We use the science of Mind Genomics, a new science which focuses on how we respond to the world of the everyday, what are the important aspects of an aspect of the everyday, and the discovery of basic groups within this this limited aspect, akin to color primaries, red blue, and yellow, respectively. Mind Genomics works with a circumscribed topic, here selling vitamin D3. Mind Genomics prescribes a standard set of activities, beginning with developing ideas and moving to the analysis of experimentally designed vignettes, and finally to the deconstruction of these vignettes into the contributions of the individual elements.

When one begins a Mind Genomics study, it often comes as a surprise to realize that there is a world of interesting information 
to be gained from the evaluation of the everyday. For example, as we move through our analysis of responses to vitamin D3, we will deal with messages, elements which are brand names, other elements which talk about consumer benefits, and still other elements which present price. As we present the elements, it will become clear that this chapter, is about selling, and not about the grand issues in the world of consumer health.

A companion paper to the present one, dealing with sleep aids [6], presents the analytical approach in detail. The reader is referred to that paper for introductions and explications of the various statistical analyses.

We begin our efforts with the introductory page, the page that respondents saw when they began the study. The respondents, 304 individuals, recruited by a field service specializing in providing panelists to take surveys, were invited to participate by means of an email. Not all people receiving such invitations respond; in fact, in general the response rate is about $5 \%$ when a person receives an unsolicited invitation. The response rate is considerably higher when the individual receiving the invitation knows that he is part of a panel, has agreed to participate in the surveys, and is working towards a reward, such as points in a loyalty program, or the chance to win a sweepstakes.

Business process note: when we take into account the effort to recruit 304 respondents, we typically find that working with these panel providers, companies specializing in delivering qualified respondents, ends up being the most economical. Even more important, these panel providers enable the study to be run quickly. Other methods, often seeming to be a bit cheaper, end up being quite expensive because the study takes a very long time to field, and often one has to make do with fewer respondents than one would like.

We see the orientation page in Figure 1. The page begins by introducing the respondent to the topic, here vitamin D3 as a wellness product. The orientation page does not provide very much information, other than telling the respondent how long the interview will be, explaining the rating questions, and telling the respondent to treat the entire screen as one single message to be rated.

Those with little experience in surveys often wonder how much information is 'just right.' In studies using the principles of Mind Genomics virtually all the key information about reactions to the ideas comes from the response to vignettes, the combinations of elements. It's important to tell the respondent to treat all the elements as one basic idea. Otherwise, without such instruction, many respondents try to rate each element separately, one at a time, and get frustrated when they can't find the rating scale for each element.

It's always a good idea not to give the respondent too much information. Giving the respondent a lengthy explanation of the topic ends up biasing the ratings. Depending upon the way the respon-

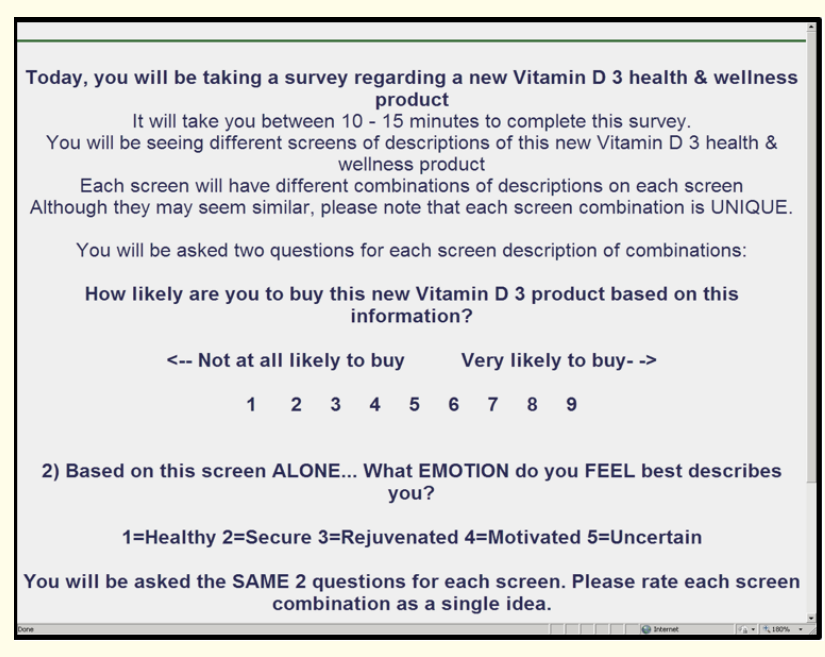

Figure 1: The orientation screen or landing page for the vitamin D3 study using Mind Genomics.

dent feels towards the extended, fact-filled orientation, the ratings may be elevated, so they bump against the top of the scale, or they may be depressed, so they bump against the bottom of the scale. We're talking here of responses to question \#1, dealing with likelihood to buy the vitamin D3 product.

\section{The test stimuli}

The heart of the Mind Genomics approach lies in the systematic variation of the messages. This study focuses on identifying the specific sales messages for vitamin D3. By sales messages we mean brand, benefit to the consumer, and price. The focus on sales comes from the objective of the project - identify 'what works' in a short sales piece, to be put on the Internet. This almost laser-like focus on sales differs from many previously executed Mind Genomics studies, where the focus was on what was important in a topic, such as what aspects of a health-care regimen were felt to be attainable for the respondent. Bringing the same power to a sales effort, rather than to a knowledge-building effort means simply changing the nature of the elements.

We see the structure of the silos and elements in Table 1. Mind Genomics works by mixing and matching together elements to create small vignettes, an example of which appears in Figure To ensure that the elements do not contradict each other, Mind Genomics begins with a bookkeeping device, elements put into larger groups known as silos. Table 1 shows us six silos, each having six elements. This particular array, called i a 6x6 design, allows us to test 36 elements, a generous number, using 48 combinations or vignettes, a number which ends up being reasonably easier for the respondent, rather than being onerous.

The elements, and indeed the silos themselves, can come from anywhere, as long as they are relevant. In this particular case, the focus of the actual project was on selling vitamin D3, so the silos 


\begin{tabular}{|c|c|}
\hline & Silo A - General Brand \\
\hline A1 & Pure Chemistry \\
\hline $\mathrm{A} 2$ & Nature's Pioneer \\
\hline A3 & Super Ecology \\
\hline A4 & Nature's IQ \\
\hline A5 & Beyond Eden \\
\hline \multirow[t]{2}{*}{ A6 } & Pure Genomics \\
\hline & Silo B - Product name \\
\hline B1 & D3 Life Spray \\
\hline B2 & Vitamin D3 Advanced \\
\hline B3 & Vitamin D3 - 5000 \\
\hline B4 & Vitamin D3 Sun Substitute \\
\hline B5 & Total D3 \\
\hline \multirow[t]{2}{*}{ B6 } & D3 Max \\
\hline & Silo C - General benefit to the consumer \\
\hline $\mathrm{C} 1$ & One spray provides more D3 than a gallon of milk \\
\hline $\mathrm{C} 2$ & $\begin{array}{l}\text { One spray provides more D3 than a day at the } \\
\text { beach }\end{array}$ \\
\hline C3 & Nature's key nutrient \\
\hline $\mathrm{C} 4$ & The complete answer to D3 deficiency \\
\hline $\mathrm{C} 5$ & Perfect for all ages \\
\hline \multirow[t]{2}{*}{ C6 } & The Sunshine Vitamin \\
\hline & Silo D - The experience \\
\hline D1 & Convenient spray formula... nothing to swallow \\
\hline D2 & Pleasant citrus taste \\
\hline D3 & Maximum strength for optimal health \\
\hline D4 & Instantly absorbed \\
\hline D5 & Orange lemon zest flavor \\
\hline \multirow[t]{2}{*}{ D6 } & Rapid sublingual absorption \\
\hline & Silo E - Health benefit \\
\hline E1 & The most bio-available form of D3 \\
\hline E2 & All natural D3 sun substitute \\
\hline E3 & Physician recommended \\
\hline E4 & Super cell health formula \\
\hline E5 & Anti-oxidant support \\
\hline \multirow[t]{2}{*}{ E6 } & 5000 IU's per spray \\
\hline & Silo F - Price \\
\hline F1 & $\$ 9.99$ for a 1-month supply \\
\hline $\mathrm{F} 2$ & $\$ 14.99$ for a 1-month supply \\
\hline F3 & $\$ 19.99$ for a 1-month supply \\
\hline $\mathrm{F} 4$ & $\$ 21.99$ for a 1-month supply \\
\hline F5 & $\$ 24.99$ for a 1-month supply \\
\hline F6 & $\$ 29.99$ for a 1-month supply \\
\hline
\end{tabular}

Table 1: The six silos and the six elements per silo for the Mind Genomics study on vitamin D3.

and elements had to be relevant to a sales situation, e.g., an advertisement in a magazine or on the web. Thus, the elements in Table 1 are short, relevant to an advertisement geared to sell, and cover the important information that one needs to have to make the sale; brand, benefits, and price.
Mind Genomics works by presenting the basic ideas (elements), combined into vignettes, or test concepts, such as we see in Figure. The vignettes present the basic ideas in a simplified format, with each element comprising a line, and each element centered, one element atop the other. Although we are accustomed to reading paragraphs of text with the elements incorporated into sentences, the format in Figure 2 makes the task easier, the interview less onerous. The respondents can simply scan the vignette and then assign a rating. The assumption is that in everyday life, people read by grazing, looking around, and taking in information, but not in the linear fashion dictated by a formal sentence structure. A purist might disagree, feeling that the vignette ought to have connectives to make it a paragraph, but the practical consideration of making the interview easy is the more important.

Figure 2 show the rating scale at the bottom of the figure. The respondent reads the vignette, and then assigns the rating. When the number corresponding to the answer has been typed (always a one-digit number), the vignette remains but the rating scale is replaced by question \#2, the selection of a feeling/emotion. The respondent once again merely selects the rating, and the vignette changes, with the computer program advancing to the next vignette.

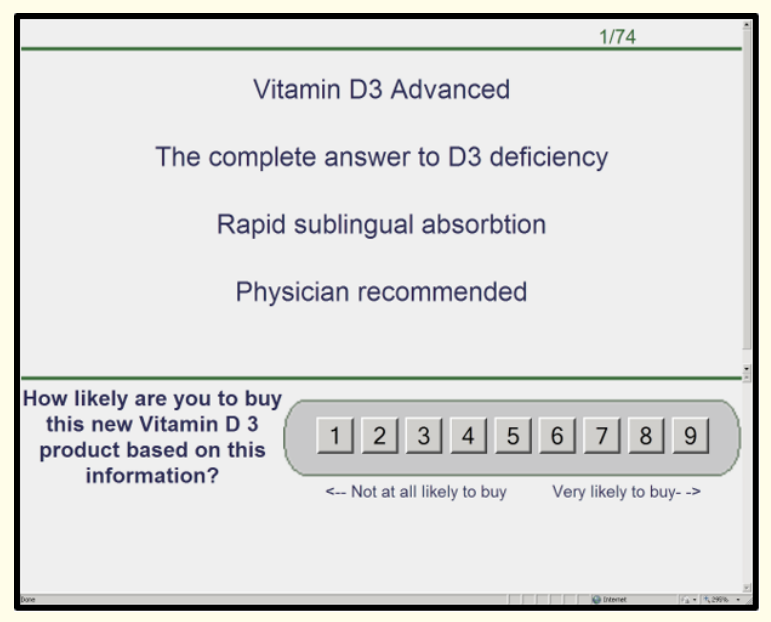

Figure 2: A four-element vignette, showing the elements, the rating scale (bottom), and the progress through the interview (top right).

At the top of the vignette, on the right-hand side we see a number, $1 / 74$. This number tells the respondent the progress. $1 / 74$ means the first screen has been completed. Once the respondent has rated the vignette on question \#1 (likelihood to buy), and question \#2 (select feeling/emotion), the number changes to 2/74 (Figure 3).

Although one might test single elements from Table 1, the elements as they are constituted really should be put together into selling propositions in Mind Genomics in order to be meaningful 


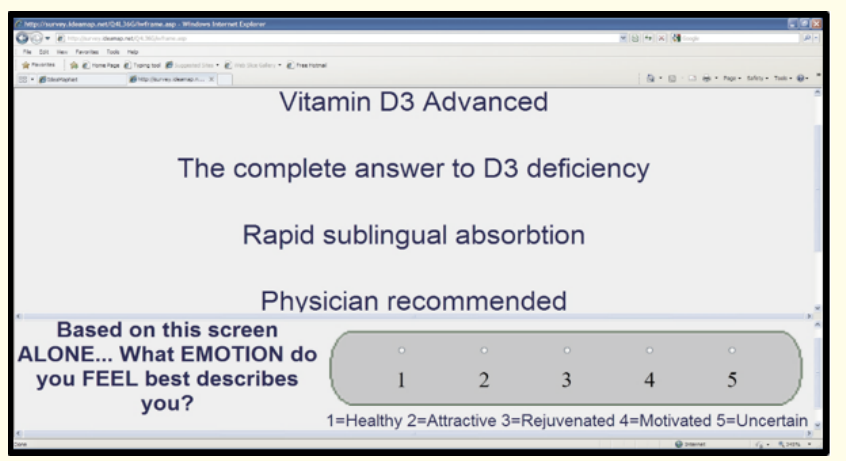

Figure 3: A four-element vignette, showing the element and the selections of emotions.

to a respondent. Presenting a respondent with single ideas, e.g., brand name or benefit or price, will generate a rating of that single idea, but the reality is that the rating is forced, and artificial. For example, giving a respondent a brand name and asking for a rating of purchase intent means that the respondent himself must conjure up the rest of the offering. It makes more sense to create combinations that simulate the more typical sales offer. And thus, we see the vignette in Figure 1. The vignette is certainly not 'complete,' but yet provides sufficient information to paint a picture, that allows a respondent to respond to an almost-meaningful offer. At the end of the day, the vignette in Figure 1 does not make the respondent feel that the information is just too sparse. There is enough information to make the judgment seem reasonable.

\section{Constructing the vignettes by experimental design}

The four elements in Figure 2 are put together according to an experimental design. The design ensures that each element appears an equal number of times, and that the elements are statistically independent, i.e., that each element acts and an independent agent.

Statisticians have developed various designs, i.e., sets of prescribed combinations, almost like recipes. For this particular study with 36 elements, the design calls for 48 vignettes, each vignette comprising 3-4 elements, each element appearing five times in the set of 48 vignettes. Experimental designs do not care which elements appear together; they simply prescribe the combinations. They need no information about what the elements happen to be, unless one wants to impose 'constraints.'. One may create a set of combinations such that certain elements are forced to appear together or are prohibited from ever appearing together We're not going to deal with these 'constrained' designs, other than to mention them here. The constraints can be accommodated, however, but the design structures will be far fewer.

The experimental design for this instantiation of Mind Genomics, the $6 \times 6$, specifies 48 combinations or vignettes. Each element appears five times. There is a significant benefit to this type of design, wherein an element appears many times. The benefit is called 'internal replication.' Rather than relying upon the response to the element based on one appearance of the element in the set of test stimuli, the experimental design forces the element to compete in five different vignettes. As a result, our estimation of how strongly an element performs is based upon a sample of five performances, against five different backgrounds. Averaging these five performances provides us with a more robust estimate of how well an element actually performs. Any element which ends up performing well across the five appearances and across many respondents is likely to be a strong performer. The design reduces the chances of an element doing well or poorly, simply 'by accident.'

Mind Genomics offers another benefit beyond the internal replication. Mind Genomics 'permutes' the experimental design, creating 'isomorphs' of the design, combinations that are mathematically the same in term of the basic structure, but combinations that are effectively 'new.' The stratagem for permuting the basic design to come up with the isomorphs has already been discussed both in the scientific literature [7] and in the US patent literature [8].

What do respondents think about these sales vignettes? (Figures 3 and 4)

A continuing theme in Mind Genomics is the nature of the response to the systematically varied vignettes. We may analyze the ratings from at least two perspectives, superficial and profound. We begin with the superficial analysis, looking at the nature of the ratings assigned to the vignettes. We end with the profound, what elements are doing to drive the ratings.

Our first analysis looks at the distribution of the ratings on the 9-point scale, and then the distribution of feelings/emotions selected. Mind Genomics provides us with a generous amount of data for our analysis. With 48 vignettes per respondent, and with 304 respondents, we are looking at 48x304 or 14,592 ratings. Figure 4 shows us how these ratings distribute on the 9-point scale. The figure suggests that the distribution is fairly flat, skewed a bit towards lower acceptance. What's important here is that the basic selling

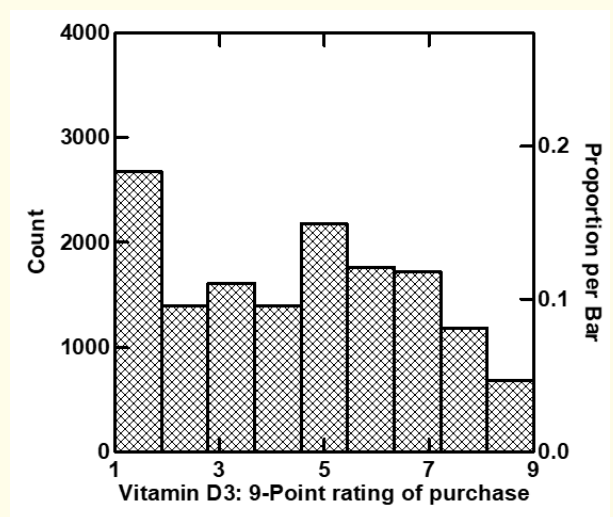

Figure 4: Distribution of purchase intent ratings for the vitamin D3 product, showing that almost a third of the respondents are quite interested in purchasing the product (ratings 7-9). 


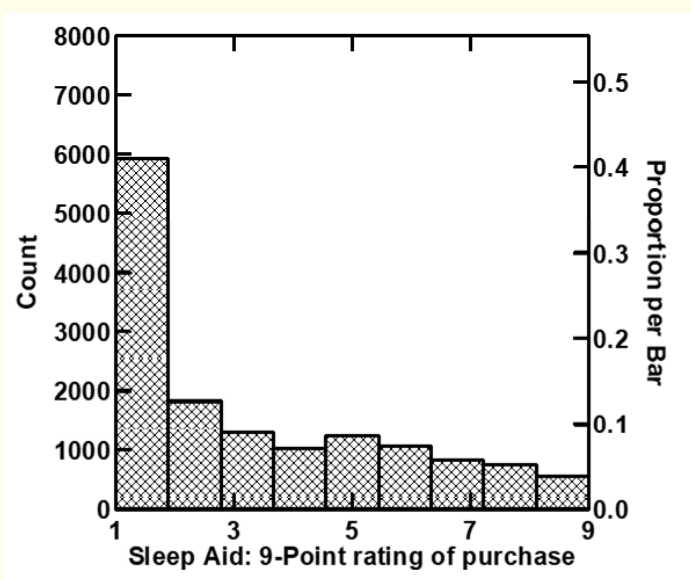

Figure 5: Distribution of purchase intent ratings for a sleep-aid product, showing that relatively few of the respondents are quite interested in purchasing the product (ratings 7-9).

messages seem to be reasonably compelling. In contrast, the distribution of ratings for sleep aids suggested far less acceptance [6].

\section{Emotions and buying interest - a macro view (Table 2)}

Each respondent evaluated 48 vignettes, selecting for each vignette one of five feelings/emotions from a list. These were healthy, secure, rejuvenated, motivated, and uncertain, respectively. One can pick many more feelings/emotions, but the requirement of the study is to have an array of such feelings/emotions, but not too many. Only by limiting to the number to say 5-7 can we uncover robust, general patterns across the entire population of 304 respondents, as well as patterns within a single respondent, based upon 48 vignettes.

When we look at the distribution of selections (bottom of Table 2 , column percents, column marked total), we see that the respondents select the feelings/emotions in different proportions. The most commonly selected feeling is 'uncertain' with $38 \%$ of the selections, the least frequently selected is 'secure' with only 8 percent of the respondents selecting.

There is an association between the feeling/emotion selected and the rating of purchase intent (question \#1). In order to establish the association, we tabulate the 9-point ratings as columns, and the feelings/emotions as rows. With nine rating points for purchase the data are too granular. To reduce granularity and in turn to let patterns emerge, we create three regions of the scale, 1-3 = low purchase interest, 4-6 = moderate purchase interest, and 7-9 = high purchase interest.

The pattern from nearly 15,000 vignettes, shown in Table 2, suggests these associations of feelings/emotions with ratings of purchase interest.
1. The data reveal slightly more low and medium purchase likelihoods, and fewer high purchase likelihoods

2. The feeling 'uncertain' is generally connected with a low purchase intent rating, a not unexpected finding

3. The feeling 'healthy' is associated with all three groups, i.e., low, medium and high likelihood of purchase. A person who says he feels healthy is not easily categorized as a likely buyer vs a likely rejecter.

4. A person who feels 'rejuvenated' is likely to express a medium purchase likelihood

5. A person who feels 'secure' is likely to express a medium or a high purchase likelihood.

6. A person who feels 'motivated' is also likely to express a medium or high purchase likelihood

We change our direction to study what respondents choose as feelings/emotions after they expressed their likelihood to purchase. These data appear at the bottom of Table 2. Look at the columns.

1. Low purchase interest (ratings 1-3) is associated with 'uncertain'

2. Medium purchase interest (ratings 4-6) is associated with 'uncertain' and 'rejuvenated'.

3. High purchase interest (ratings 7-9) is associated with 'motivated'.

\begin{tabular}{|l|c|c|c|c|c|}
\hline \multicolumn{6}{|c|}{ Row percents - correlations with the five feelings/emotions } \\
\hline & $\begin{array}{c}\text { Low } \\
(1-3)\end{array}$ & $\begin{array}{c}\text { Med } \\
(4-6)\end{array}$ & $\begin{array}{c}\text { High } \\
(7-9)\end{array}$ & Total\% & N \\
\hline Uncertain & 67 & 28 & 6 & 100 & 5611 \\
\hline Healthy & 40 & 28 & 32 & 100 & 2592 \\
\hline Rejuvenated & 13 & 59 & 28 & 100 & 2313 \\
\hline Secure & 23 & 43 & 34 & 100 & 1282 \\
\hline Motivated & 10 & 41 & 49 & 100 & 2794 \\
\hline Total & 39 & 37 & 25 & 100 & \\
\hline N & 5680 & 5332 & 3580 & & 14592 \\
\hline & & & & & \\
\hline
\end{tabular}

Column percents - correlations with the three purchase interest regions on the 9 point scale

\begin{tabular}{|l|c|c|c|c|c|}
\hline & $\begin{array}{c}\text { Low } \\
(1-3)\end{array}$ & $\begin{array}{c}\text { Med } \\
(4-6)\end{array}$ & $\begin{array}{c}\text { High } \\
(7-9)\end{array}$ & Total & N \\
\hline Uncertain & 66 & 29 & 9 & 38 & 5611 \\
\hline Healthy & 18 & 14 & 23 & 18 & 2592 \\
\hline Rejuvenated & 5 & 26 & 18 & 16 & 2313 \\
\hline Secure & 5 & 10 & 12 & 9 & 1282 \\
\hline Motivated & 5 & 21 & 38 & 19 & 2794 \\
\hline Total & 100 & 100 & 100 & 100 & \\
\hline N & 5680 & 5332 & 3580 & & 14592 \\
\hline
\end{tabular}

Table 2: Association between question \#1 (purchase likelihood; columns) and selected feeling/emotion (rows). The row percent show the distribution of purchase likelihoods for each feeling/ emotion. The column percent show the distribution of feelings/ emotions for each purchase likelihood. 
Deconstructing vignettes by OLS (ordinary least-squares) regression

The essence of Mind Genomics is to deconstruct the response assigned to a vignette into the contributions of the elements in the vignette. A nice property of the vignette is that there are enough different messages in the vignette to prevent virtually any respondent from 'gaming' the system. That is, it's impossible for a respondent to adopt a strategy which provides a politically correct answer; too much is going on the vignette. By the same token, it's impossible to use the responses to the vignettes as direct measures of element importance. The responses are assigned to combinations, not to single elements.

Mind Genomics uses regression, or 'curve fitting' to deconstruct the response into the contributions of the different components. Regression, specifically OLS (ordinary least-squares) regression fits a simple equation to the data, with the parameters of the equation showing us how the different elements contribute to the response.

We write the general equation as follows: Rating $=\mathrm{k}_{0}+\mathrm{k}_{1}(\mathrm{~A} 1)+$ $\mathrm{k}_{2}(\mathrm{~A} 2) . . \mathrm{k}_{36}(\mathrm{~F} 6)$

The response is simply the sum of an additive constant $\left(\mathrm{k}_{0}\right)$, and weighting factors, $\mathrm{k}_{1} . \mathrm{k}_{36^{\prime}}$ applied to the elements. The elements, in turn, take on only two values, 0 when absent from a vignette, and 1 when present in a vignette, respectively.

When we look at the equation above, we cannot help but be struck by its simplicity. We have very few 'moving parts,' specifically the elements, which are absent or present, the weight attached to the element, and the additive constant or baseline. We don't have to guess about the values of the parameters, $\mathrm{k}_{0}$, and $\mathrm{k}_{1}-\mathrm{k}_{36}$. OLS regression provides a determined set of values, those values being the ones which minimize the square of differences between the actual values assigned to the vignette, and the estimated values of the rating of the vignette. That is, OLS regression identifies the one 'solution', i.e., the one set of values, $\mathrm{k}_{0}$, and $\mathrm{k}_{1}-\mathrm{k}_{36}$ which minimizes a specific 'expression,' namely the sum of squared differences, with each difference corresponding to one of the vignettes (predicted vignette rating - actually measured vignette rating).

Persuasion vs Interest: Desire to buy versus 'belonging to the buyer class'

Mind Genomics follows the world view of sociologists, and market researchers. These two groups measure the entire individual, or better, measure the proportion of individuals who belong to one of several complementary, exhaustive groups (e.g., married versus unmarried, male versus female). To sociologists and to market researchers, one's 'degree of feeling' may be of interest, but it's not the way they think of people, nor the relevant statistic which they use when discussing their data. To sociologists and market research- ers, indeed to people measuring the behavior of large groups, the nature of one's feeling is a private affair. Of course, it's interesting to count the number of people holding different points of view, but the exact strength of belief is not of interest.

Contrast this focus on group memberships with a psychologist's focus on the person himself, the degree of feeling. The psychologist may be interested in groups, but the primary focus of the psychologist is on the person himself, such as the degree of feeling, degree of purchase probability.

These two foci, group membership versus individual degree of feeling, can be accommodated by OLS regression, using the same data, albeit treated differently. OLS produces for us two different models, the Persuasion Model for intensity of feeling (psychological focus), and the Interest Model for membership in a class (e.g., acceptors of the idea presented by the vignette, the sociological focus). The key different between the two types of analysis is the way that we define the dependent variable when we do an OLS regression.

Persuasion Model: This model shows us how the different elements combine to generate the 9-point rating. OLS uses the actual 9-point ratings as the dependent variable, and the presence/absence of the 36 elements as the independent variables. When the element is present in a particular vignette, the element is assigned a value ' 1 ' in order to denote that presence. Otherwise, the absent element is assigned a value ' 0 .' The information needed for the OLS regression is straightforward and limited:

1. The 48 rows (one per vignette, per respondent)

2. The array of 0 's and 1's for each vignette.

3. With 3-4 elements present in a vignette, most of the independent variables will end up with the value 0 .

4. The actual rating assigned to the vignette

OLS estimates the additive constant and the 36 coefficients or impact values of the 36 individual elements. The equation is expressed as: 9-Point Rating $=\mathrm{k}_{0}+\mathrm{k}_{1}(\mathrm{~A} 1)+\mathrm{k}_{2}(\mathrm{~A} 2) . . \mathrm{k}_{36}(\mathrm{~F} 6)$. This is called the Persuasion Model, with the name chosen simply to refer to the model. We selected the term Persuasion Model because the equation shows the strength of each element as a driver of persuasion, with persuasion measured by the 9-point rating.

The experimental design that we use to create the 48 vignettes allows us to estimate the parameters of the Persuasion Model for each respondent, one respondent at a time. This is called a within-subjects analysis; everything we need for analysis is contained within the design and ratings assigned by a single person.

The Persuasion Model is 'granular,' because the OLS regression uses all nine rating points as possible values for the dependent variable. We get a sense of the mind of the respondent, what's impor- 
tant, what's not. We will use the Persuasion Model when we divide the respondents by the patterns of elements which drive degree of buying probability (so-called segmentation or cluster analysis.)

Interest Model: The Interest Model emerges after transforming the ratings, to convert the original 9-point rating scale to a binary, $0 / 100$ scale. The specifics of the transformation are typically left to the researcher; there are no hard and fast rules which dictate how we divide the nine points. The typical rule of thumb is to divide the scale in a way which is intuitively meaningful. For most previous studies using Mind Genomics (rule developing experimentation), the practice has been to divide the scale into a lower half (1-6) transformed to 0 , and a higher half (7-9) transformed to 100.

In some cases, e.g., when one works with populations who 'uprate' vignettes, assigning the vignettes high ratings (e.g., 7-9) out of a cultural proclivity to assign high ratings, one can make a more stringent criterion (e.g., 1-7 $\rightarrow$ 0; 8-9 $\rightarrow$ 100). This more stringent criterion is often used with work among Hispanic respondents, who tend to up-rate the vignettes, assigning a disproportionate number of vignettes ratings of 8 and 9 , respectively, i.e., ratings at the top of the scale.

Since each respondent evaluated 48 different vignettes arranged according to an experimental design, we easily estimate the parameters of the Interest Model for each respondent. There is only one intermediate step before estimating those parameters; for each the $0 / 100$ value for each vignette, for each respondent, we add a very small random number $\left(<10^{-5}\right)$. This stratagem ensures that the regression analysis will work at the level of the individual respondent, even when the respondent rated all vignettes 6 or lower (transformed to 0 ), or all vignettes 7 or higher (transformed to 100). Adding the small random number ensures that the regression analysis will work at the level of the individual respondent.

\section{What the Interest Model reveals (Table 3)}

Recall that the 'Interest' value is the binary transform of the 1-9 rating scale, with ratings of 1-6 transformed to ' 0 ' to denote little or no interest, and ratings of 7-9 transformed to ' 100 ' to denote more interest. We make this transformation because it becomes much easier to understand the results. Managers as well as regular people understand 'no/yes' more easily than they understand the notion of magnitude, although the reality is that magnitude is easier to measure. No/Yes requires an a priori decision on the part of the researcher and respondent. No/Yes is all-or-none. In the words of author HRM's late doctoral professor, S.S. Stevens, 'nothing is harder in psychological science than going from a continuous measure to a discrete measure".

We estimate the 37 parameters of the Interest Model, for each respondent, and then average the corresponding parameters. With
304 respondents, the average parameter is based on 304 observations. This base size, $300+$ respondents, allows us to feel comfortable about the what 'works' with vitamin D3, and what does not work. Table 3 presents the results, with the elements sorted in descending Mind Genomics.

Looking at the data in Table 3 tells us a great deal about what the total panel would say, i.e., the average respondent.

1. The additive constant is a low 19 , with 19 meaning that in the absence of any elements the conditional probability is only $19 \%$ that a respondent will assign a rating of 7-9 to a 'selling vignette' about vitamin D3. Most respondents are not predisposed to up-rate these sales-oriented vignettes about vitamin D3. The selling vignette not have the 'basic magic' of say a vitamin $\mathrm{C}$. It's the messages that have to do the work.

2. Low prices work (e.g., \$9.99 or \$14.99 for a 1-month supply perform well)

3. The other elements do not perform well. The highest impact value is +4 , meaning that only $4 \%$ more respondents will rate the vignette as $7-9$. Thus, combining the additive constant $(+19)$ with the best scoring elements (e.g., instantly absorbed), will bring up the impact value of 23 . We see this from the sum of the additive constant and the impact value of the element.

4. Price makes a difference. By the time we reach $\$ 24.99$ for 1-month supply we end up with a price that is perceived to be far too expensive (impact $=-6$, meaning that we lose $6 \%$ of the respondents when we raise the price to $\$ 24.99$.) Thus, were we to charge $\$ 24.99$ for a 1-month supply, and say nothing more, we would expect $19-6$ or $13 \%$ of the respondents to say that they would be interested in buying vitamin D3.

\section{Gender makes a small difference (Table 4)}

Dividing our respondents into gender suggest slight differences between the genders

1. Males are slightly more predisposed to buy vitamin D3 (additive constant is 23 for males, 16 for females).

2. Males respond strongly to anti-oxidants, females do not (8 for males, 0 for females).

3. Males respond strongly to the name Total D3, females do not ( 7 for males, 1 for females)

4. Females respond strongly to the name 'Vitamin D3 Advanced' ( 0 for males, 6 for females)

5. There are no clear differences in responses to prices; as prices increase the interest in vitamin D3 decreases

6. The bottom line is that there are differences, but they are rather few and not dramatic 


\begin{tabular}{|c|c|}
\hline $\begin{array}{l}\text { Vitamin D3 - Interest Model for the total panel } \\
\text { ( } n=304 \text { respondents) }\end{array}$ & $\begin{array}{l}\text { Total } \\
\text { Sample }\end{array}$ \\
\hline Base Size & 304 \\
\hline Additive constant & 19 \\
\hline$\$ 9.99$ for a 1-month supply & 19 \\
\hline$\$ 14.99$ for a 1-month supply & 7 \\
\hline Vitamin D3 - 5000 & 4 \\
\hline Total D3 & 4 \\
\hline Instantly absorbed & 4 \\
\hline Physician recommended & 4 \\
\hline Nature's IQ & 3 \\
\hline Vitamin D3 Advanced & 3 \\
\hline Vitamin D3 Sun Substitute & 3 \\
\hline One spray provides more D3 than a gallon of milk & 3 \\
\hline Maximum strength for optimal health & 3 \\
\hline Anti-oxidant support & 3 \\
\hline Nature's Pioneer & 2 \\
\hline One spray provides more D3 than a day at the beach & 2 \\
\hline The complete answer to D3 deficiency & 2 \\
\hline Perfect for all ages & 2 \\
\hline Pleasant citrus taste & 2 \\
\hline All natural D3 sun substitute & 2 \\
\hline Pure Chemistry & 1 \\
\hline Beyond Eden & 1 \\
\hline D3 Max & 1 \\
\hline Convenient spray formula... nothing to swallow & 1 \\
\hline Orange lemon zest flavor & 1 \\
\hline The most bio-available form of D3 & 1 \\
\hline 5000 IU's per spray & 1 \\
\hline$\$ 19.99$ for a $1-$ month supply & 1 \\
\hline Nature's key nutrient & 0 \\
\hline The Sunshine Vitamin & 0 \\
\hline Rapid sublingual absorption & 0 \\
\hline Super cell health formula & -1 \\
\hline Super Ecology & -2 \\
\hline Pure Genomics & -2 \\
\hline D3 Life Spray & -2 \\
\hline$\$ 24.99$ for a 1 -month supply & -6 \\
\hline$\$ 21.99$ for a 1-month supply & -7 \\
\hline$\$ 29.99$ for a 1 -month supply & -9 \\
\hline
\end{tabular}

Table 3: The additive constant (basic interest) and the impact values of the 36 elements, for vitamin D3.

Age also makes a difference, but not a big one (Table 5)

As respondents get older they respond differently to the vitamin D3 product:

1. The older respondents (age 53+) are really disinterested in the basic idea of vitamin D3. The additive constant is 10 , meaning that only $10 \%$ of the respondents age $53+$ would

\begin{tabular}{|l|c|c|}
\hline & Males & Females \\
\hline Base size & 133 & 171 \\
\hline Additive constant & 23 & 16 \\
\hline Winning elements - males & & \\
\hline Anti-oxidant support & 8 & 0 \\
\hline Total D3 & 7 & 1 \\
\hline Physician recommended & 6 & 3 \\
\hline & & \\
\hline Winning elements - females & & \\
\hline Vitamin D3 Advanced & 0 & 6 \\
\hline & & \\
\hline Pricing & & \\
\hline \$9.99 for a 1-month supply & 18 & 19 \\
\hline \$14.99 for a 1-month supply & 5 & 9 \\
\hline \$19.99 for a 1-month supply & -2 & 3 \\
\hline \$21.99 for a 1-month supply & -6 & -7 \\
\hline \$24.99 for a 1-month supply & -7 & -5 \\
\hline \$29.99 for a 1-month supply & -8 & -10 \\
\hline
\end{tabular}

Table 4: Winning elements and pricing results for Vitamin D3, by gender.

assign a rating of 7-9 to a vignette about vitamin D3. It's the messages which must to do the work.

2. The younger respondents, younger than 39 years, want to know that the product has a good citrus taste, and is both physician recommended, as well as maximum strength (impacts of 8,7 and 7, respectively).

3. The middle level, ages $35-52$ change their focus, responding to anti-oxidants (impact $=7$ ).

4. The oldest respondents, age 53+, respondent only to one name, Vitamin D3 Advanced.

5. In terms of pricing, the three age groups of respondents show similar, but not identical patterns. The oldest group respondents slightly more strongly when the price plummets to $\$ 9.99$ for a 1-month supply. The impact of this low price is +22 for the older respondents, and +17 for the younger respondents

6. The bottom line is similar to what we saw for gender, namely slight effects at best.

\section{Mind-set segmentation (Table 6)}

Mind Genomics often reveals the existence of groups of respondents showing moderately and occasionally radically different patterns of sensitivities to elements. These groups of respondents, mind-set segments, respond to different themes. The individuals in a segment may seem dissimilar to each other; a segment might comprise males and females, as well as individuals monitoring different health issues, and using different products. That is, the segment is heterogeneous with respect to many of the measures that a marketer feels to be important in classifying a person. Yet these segments are compelling. 


\begin{tabular}{|l|l|l|l|}
\hline & Age $<39$ & Age 39-52 & Age $\mathbf{5 3}$ \\
\hline Base Size & 82 & 105 & 117 \\
\hline Additive constant & 24 & 25 & 10 \\
\hline Age <39 years & & & \\
\hline Pleasant citrus taste & 8 & -2 & 0 \\
\hline Physician recommended & 7 & 6 & 0 \\
\hline $\begin{array}{l}\text { Maximum strength for } \\
\text { optimal health }\end{array}$ & 7 & 2 & 1 \\
\hline Age 39-52 & & & \\
\hline Vitamin D3 - 5000 & -1 & 8 & 4 \\
\hline Anti-oxidant support & 0 & 7 & 2 \\
\hline Age 53+ & & & \\
\hline Vitamin D3 Advanced & 1 & 0 & 8 \\
\hline & & & \\
\hline & & & \\
\hline \$9.99 for a 1-month supply & 17 & 17 & 22 \\
\hline \$14.99 for a 1-month supply & 4 & 11 & 7 \\
\hline \$19.99 for a 1-month supply & 1 & -2 & 3 \\
\hline \$21.99 for a 1-month supply & -7 & -9 & -4 \\
\hline \$24.99 for a 1-month supply & -8 & -5 & -5 \\
\hline \$29.99 for a 1-month supply & -12 & -11 & -6 \\
\hline & & & \\
\hline
\end{tabular}

Table 5: Winning elements and pricing results for Vitamin D3, by age.

We divide the respondents by the pattern of impact values, using the statistical method of clustering, and using the impact values from the Persuasion Model. (We could just as easily divide the respondents by the pattern of their impact values from the Interest Model.) The statistical procedures, cluster analysis, does not tell us the nature of the segments, nor whether we should divide the population of respondents into two segments, three segments, or four segments. The number of segments and the nature of the segments is left to interpretation; it is more of an art than a science when it comes to interpretation. In mind-set segmentation we aim for parsimony (fewest number of segments), and interpretability (the segments mean something).

When we apply the segmentation algorithm to the data for vitamin D3, we end up with hree 'interpretable' groups of respondents, as Table 6 shows

1. Segment 1 , the largest group comprises $2 / 3$ of the respondents, with a base size of 210 , i.e., about $2 / 3$, responds to general statements, such as physician recommended, or all natural D3 sun substitute. The impact values are fairly low, however, the highest being +7 .

2. Segment 2 with 49 respondents, $1 / 6$ of the group, responds to messages about product efficacy.

3. Segment 3, with 45 respondents, also $1 / 6$ of the group, responds to the names. Segment 3 starts at no interest at all (additive constant 0 ), but the elements, especially names, generate interest.

4. In terms of prices, as price increases, interest in the product decreases quite dramatically.

5. Segment 3, which responds to names, responds most strongly and positively to low prices.

6. Summing up, for vitamin D3, not many elements work well. It's a matter of pricing, but the elements do not spark attention, perhaps because respondents do not really know what vitamin D3 does.

\begin{tabular}{|c|c|c|c|}
\hline & Seg1 & Seg2 & Seg 3 \\
\hline Base size & 210 & 49 & 45 \\
\hline Additive constant & 22 & 25 & 0 \\
\hline \multicolumn{4}{|l|}{ Segment 1 - General } \\
\hline Physician recommended & 7 & -5 & 1 \\
\hline All natural D3 sun substitute & 7 & -16 & 0 \\
\hline \multicolumn{4}{|l|}{ Segment 2 - Responds to efficacy } \\
\hline Instantly absorbed & 3 & 10 & 0 \\
\hline Vitamin D3 - 5000 & 2 & 8 & 12 \\
\hline $\begin{array}{l}\text { One spray provides more D3 than a } \\
\text { gallon of milk }\end{array}$ & -1 & 8 & 15 \\
\hline $\begin{array}{l}\text { One spray provides more D3 than a day } \\
\text { at the beach }\end{array}$ & -1 & 8 & 11 \\
\hline \multicolumn{4}{|l|}{ Segment 3 - Responds to names } \\
\hline Super Ecology & -4 & -11 & 17 \\
\hline Nature's key nutrient & -3 & -1 & 17 \\
\hline The complete answer to D3 deficiency & -1 & 0 & 16 \\
\hline Pure Chemistry & 0 & -8 & 16 \\
\hline Nature's IQ & 3 & -9 & 15 \\
\hline $\begin{array}{l}\text { One spray provides more D3 than a } \\
\text { gallon of milk }\end{array}$ & -1 & 8 & 15 \\
\hline Vitamin D3 - 5000 & 2 & 8 & 12 \\
\hline Perfect for all ages & -1 & 3 & 11 \\
\hline Pure Genomics & -2 & -13 & 11 \\
\hline $\begin{array}{l}\text { One spray provides more D3 than a day } \\
\text { at the beach }\end{array}$ & -1 & 8 & 11 \\
\hline Vitamin D3 Advanced & 3 & -1 & 11 \\
\hline Nature's Pioneer & 2 & -5 & 10 \\
\hline Maximum strength for optimal health & 2 & 4 & 9 \\
\hline Beyond Eden & 2 & -9 & 8 \\
\hline \multicolumn{4}{|l|}{ Prices } \\
\hline$\$ 9.99$ for a 1-month supply & 16 & 17 & 36 \\
\hline$\$ 14.99$ for a 1-month supply & 2 & 13 & 27 \\
\hline$\$ 19.99$ for a 1-month supply & -6 & 5 & 28 \\
\hline$\$ 21.99$ for a 1-month supply & -12 & -6 & 18 \\
\hline$\$ 24.99$ for a 1 -month supply & -10 & -3 & 11 \\
\hline$\$ 29.99$ for a 1 -month supply & -15 & -4 & 11 \\
\hline
\end{tabular}

Table 6: Winning elements and pricing results for Vitamin D3, by three mind-set segments. 


\section{What emotions link with the elements (Table 7)}

Table 2 showed us the proportion of times that respondents selected each of the five feelings/emotions. Recall that the respondent was required to selected exactly one of five feelings/emotions.

OLS regression enables us to link the presence of an element to the selection of each of the five feelings/emotions. These feelings/ emotions do not lie on a scale. Rather, they are presumed to reflect five different feelings, four of which are positive (healthy, attractive, rejuvenated, motivated) and one of which is negative (uncertain). The five feelings/emotions are not meant as a scale, but rather as the 'coloration' or 'tonality' of the element.

We relate the feelings/emotions to the elements by a simple statistical stratagem. We create five new 'dummy' variables to show the selection, and then do the analysis, following the steps below. These steps move us from a selection of feelings/emotions to the linkage of each with the elements.

1. Create five new dummy variables, one each for feeling/emotion; uncertain, healthy, attractive, rejuvenated, and motivated, respectively. Were we to be dealing with seven feelings/emotions, not five, we would create seven new dummy variables, not five.

2. For each vignette identify the feeling/emotion selected, and put in the value 100 , plus a small random number $(<10-5)$. For the remaining four feelings/emotions that were not selected, we put in the value 0 plus a small random number (again < 10-5).

3. We now have transformed our feelings/emotions into five scales.

4. For each feeling/emotion, we now relate this specific feeling/emotion to the presence/absence of the 36 elements. Our tool will be OLS (ordinary least-squares) regression, the same workhorse tool that we have used.

5. We will not use the additive constant. We assume that when there are no elements, there is absolutely no way we could say that there is an emotion tied with the topic - at least that is what we are assuming here.

6. To make the analysis simple we put all of the data into one large file. The first column of the data file comprises the respondent ID number. The second set of numbers (columns $2-37)$ comprise the binary values of the 36 elements $(0=a b$ sent, $1=$ present). The third set, really one number, column (38) shows which of the five feelings/emotions was selected for that vignette. The fourth set of numbers (columns 39-43) comprise the recoded responses $(0+$ small random number when the feeling/emotion as not chosen; $100+$ small random number when the feeling/emotion was chosen).

7. Our revision of the data to five new dependent variables now enables us to relate the presence/absence of the 36 elements to the feeling/emotion selected.
8. The coefficients or linkage values tell the whole story (Table 7). Each element in the vignette brings some feeling/emotion. The numbers in the body of Table 7 show the percent of times a specific emotion can be linked to the element, when the element is one of an average of 3.75 elements in a vignette.

9. To get a sense of how an element would link with an emotion in a 1-element vignette, just multiply all of the linkages in Table 7 by 3.75 . That product, e.g., $15 \times 3.75=56.25$ is the approximately percent of the time that the element, e.g. the name 'Pure Genomics' as a single element would be linked with the feeling 'uncertain.'

10. Now that we have the linkages, Table 7 tells us that the majority of the feelings/emotions are 'uncertain.' Only one element, the name Vitamin D - 3000, links to a positive feeling, this time 'healthy'.

11. The bottom line here is that by probing another dimension, namely feelings/emotions, we begin to get a sense of how the respondent feels, and just what type of feeling is engendered by the messaging. It is one thing to identify winning elements, simply by the impact values of those elements in a vignette. It is an entirely different thing to get a sense of the emotions that the elements create. For example, these four elements scores equally well on the Interest Model, but only the name, Vitamin D3 - 5000, generates a feeling of 'healthy':

Vitamin D3 - 5000

Total D3

Instantly absorbed

Physician recommended

\section{Do feelings/emotions link with price (Table 8)?}

Part of the Mind Genomics exercise for vitamin D3 was the offer of one-month supplies. We know from Table 3 that respondent ratings of interest in buying the vitamin decreases with increasing price. Even though the respondent never saw two prices together in the same vignette, the respondents were sensitive to price; the increase in price acted as a brake on the purchase interest. This finding alone is face-validity that the respondents are acting in a reasonable fashion.

What happens to the selection of feelings/emotions when we present the respondents with the different prices? For example, when the price is very low, $\$ 9.99 /$ month, what do respondents say they feel? Table 8 tells us that the respondents say that they feel motivated (linkage $=11$ ). As we raise the price the respondents say that feel increasingly uncertain. The other feelings/emotions are virtually irrelevant.

Can we segment respondents by the pattern of their emotions (Table 9)?

Up to now we have seen that our response patterns are fairly flat when we instruct the respondent to tell us how interested he 


\begin{tabular}{|l|c|c|c|c|c|}
\hline \multicolumn{1}{|c|}{ Total Sample } & Uncertain & Healthy & Attractive & Rejuvenated & Motivated \\
\hline Pure Genomics & 15 & 2 & 2 & 4 & 4 \\
\hline Nature's Pioneer & 14 & 5 & 1 & 3 & 6 \\
\hline Super Ecology & 14 & 4 & 0 & 5 & 6 \\
\hline Rapid sublingual absorption & 14 & 3 & 4 & 2 & 5 \\
\hline Pure Chemistry & 13 & 4 & 1 & 5 & 6 \\
\hline Nature's key nutrient & 13 & 4 & 1 & 3 & 6 \\
\hline \$19.99 for a 1-month supply & 13 & 2 & 6 & 4 & 3 \\
\hline Nature's IQ & 12 & 5 & 2 & 4 & 4 \\
\hline Beyond Eden & 12 & 4 & 0 & 8 & 3 \\
\hline D3 Life Spray & 11 & 5 & 3 & 5 & 4 \\
\hline D3 Max & 11 & 3 & 3 & 5 & 4 \\
\hline Perfect for all ages & 11 & 4 & 1 & 4 & 6 \\
\hline Total D3 & 10 & 4 & 4 & 5 & 3 \\
\hline Convenient spray formula. nothing to swallow & 10 & 4 & 3 & 2 & 6 \\
\hline Orange lemon zest flavor & 10 & 2 & 2 & 8 & 5 \\
\hline Super cell health formula & 10 & 6 & 3 & 5 & 3 \\
\hline Vitamin D3 - 5000 & 4 & 10 & 3 & 2 & 7 \\
\hline
\end{tabular}

Table 7: How elements links with feelings/emotions. The numbers in the body of the table show the percent of times that an element would be linked with the feeling/emotion shown in the column, when the element appears in a vignette comprising 3.75 elements. Random linkages are values 5-6. Linkages of 10 or more are significant.

\begin{tabular}{|l|c|c|c|c|c|c|}
\hline & Total Sample & Uncertain & Healthy & Attractive & Rejuvenated & Motivated \\
\hline F1 & \$9.99 for a 1-month supply & 3 & 5 & 2 & 4 & $\mathbf{1 1}$ \\
\hline F2 & \$14.99 for a 1-month supply & 9 & 4 & 3 & 5 & 6 \\
\hline F4 & \$21.99 for a 1-month supply & 16 & 4 & 3 & 2 & 2 \\
\hline F5 & \$24.99 for a 1-month supply & 20 & 3 & 2 & 4 & -2 \\
\hline F6 & \$29.99 for a 1-month supply & 21 & 3 & 1 & 2 & -1 \\
\hline
\end{tabular}

Table 8: Linkage between price per month for vitamin D3 and the selection of a feeling/emotion.

is in purchasing the vitamin D3 product. Furthermore, the primary feeling/emotion is 'uncertain'.

Do we find any differences in feeling/emotion and even in purchase intent (question \#1) when we divide our respondents by the patterns of the emotions which emerged when the respondents evaluated their 24 different vignettes. Each respondent generated five sets of 36 numbers each, or a total of 180 numbers. Each of the five sets of numbers corresponds to one of the five feelings/emotions. Each set of 36 numbers within the five sets corresponds to the linkage between the specific feeling/emotion and the specific element.

By clustering respondents based upon the 180 numbers we identify different groups of respondents, groups who differ in the pattern of feelings/emotions that they select for the different elements. Table 9 shows us the results from creating two segments, the segment first comprising 152 respondents and the second seg- ment comprising 88 respondents. The segments were created by using Systat's K-Means program, with the Pearson R (correlation) as the basic statistic, and the value $(1-$ Pearson $R)$ as the measure of distance between any two respondents.

Table 9 suggests that:

1. Segment 1 chooses the feeling/emotion 'uncertain most of the time. The other feelings/emotions are virtually irrelevant.

2. Segment 2 selects 'motivated' and 'rejuvenated' most of the time, and only rarely does Segment 2 select 'uncertain.' Segment 2 is certainly more positive to the messages, at least in terms of what they say they feel after reading a vignette.

3. We do not know as yet whether the more positive affect shown by Segment 2, i.e., those respondents selecting motivated and rejuvenated, will translate into more interest in buying the Vitamin D3 product. That analysis now follows. 


\begin{tabular}{|c|c|c|}
\hline & $\begin{array}{c}\text { Emotion Segment } 1 \\
\mathrm{~N}=152 \\
\text { (uncertain) }\end{array}$ & $\begin{array}{c}\text { Emotion Segment } 2 \\
\mathrm{~N}=99 \\
\text { (motivated, rejuvenated) }\end{array}$ \\
\hline & \multicolumn{2}{|c|}{$\begin{array}{l}\text { Percent of time the feeling/emotion was } \\
\text { selected }\end{array}$} \\
\hline Uncertain & 68 & 8 \\
\hline Motivated & 14 & 36 \\
\hline Rejuvenated & 4 & 38 \\
\hline Secure & 3 & 14 \\
\hline Healthy & 10 & 5 \\
\hline Total & 100 & 100 \\
\hline
\end{tabular}

Table 9: Segmentation of the respondents into group, based upon the linkage pattern between element and feelings/emotions. The table shows the frequency of times respondents in each segment select each of the five feelings/emotions. Segment 1 choose uncertain. Segment 2 chooses motivated and rejuvenated.

How emotion segments respond to elements (Table 10)

An ingoing hypothesis for emotion-response segmentation is that the segments responding with positive selections (e.g., motivated, rejuvenated) would be more interested in buying the vitamin D3 product. The underlying reasoning is that a happy customer is a customer likely to buy. The data in Table 10 supports that hypothesis, but in an unusual way.

1. The additive constants differ dramatically different. For Emotion Segment 1, distinguished by their selection of 'uncertain,' the additive constant is

2. Yet there are a number of elements which perform well for Emotion Segment 1, mainly low price and reassurance

3. For Emotion Segment 2, those who selected motivated and rejuvenated, we see a much higher additive constant, 35 . More than a third of these respondents will buy without any elements. Add a low price, and the number goes to 51 , more than half.

4. No other elements work.

5. The bottom line here is that segmenting respondents by their emotions shows radically different mind-sets with respect both to the emotions that they select, and they way that they make their decisions. Yet, in terms of the strong performing elements, few elements perform well.

\section{Summing up}

In most Mind Genomics studies, the elements in the study deal with positive or occasionally negative features of a product or situation. The features are generally 'rich' in terms of what they convey; positive aspects of the product or situation that are designed to benefit the respondent. In those typical studies the strongest performing elements tend to often are often quite 'rich,' dealing as they do with substantive benefits to the respondent.

\begin{tabular}{|c|c|c|c|}
\hline Effect & & $\begin{array}{c}\text { Emotion } \\
\text { Seg1 } \\
\text { Uncertain }\end{array}$ & $\begin{array}{c}\text { Emotion Seg2 } \\
\text { Motivated } \\
\text { Rejuvenated }\end{array}$ \\
\hline & Additive constant & 2 & 35 \\
\hline & $\begin{array}{l}\text { Emotion Segment } 1 \text { - } \\
\text { Uncertain }\end{array}$ & & \\
\hline $\mathrm{F} 1$ & $\begin{array}{l}\$ 9.99 \text { for a } 1 \text {-month } \\
\text { supply }\end{array}$ & 19 & 16 \\
\hline B3 & Vitamin D3 - 5000 & 9 & 2 \\
\hline F2 & $\begin{array}{l}\$ 14.99 \text { for a 1-month } \\
\text { supply }\end{array}$ & 9 & -1 \\
\hline $\mathrm{C} 4$ & $\begin{array}{l}\text { The complete answer to } \\
\text { D3 deficiency }\end{array}$ & 8 & -1 \\
\hline D3 & $\begin{array}{l}\text { Maximum strength for } \\
\text { optimal health }\end{array}$ & 8 & 3 \\
\hline $\mathrm{D} 2$ & Pleasant citrus taste & 7 & -1 \\
\hline E3 & Physician recommended & 7 & 3 \\
\hline \multirow[t]{2}{*}{ F6 } & $\begin{array}{l}\$ 29.99 \text { for a 1-month } \\
\text { supply }\end{array}$ & -6 & -13 \\
\hline & $\begin{array}{l}\text { Emotion Segment } \\
2 \text { - Rejuvenated and } \\
\text { motivated }\end{array}$ & & \\
\hline $\mathrm{F} 1$ & $\begin{array}{l}\$ 9.99 \text { for a } 1 \text {-month } \\
\text { supply }\end{array}$ & 19 & 16 \\
\hline $\mathrm{F} 4$ & $\begin{array}{l}\$ 21.99 \text { for a } 1 \text {-month } \\
\text { supply }\end{array}$ & -3 & -9 \\
\hline F5 & $\begin{array}{l}\$ 24.99 \text { for a } 1 \text {-month } \\
\text { supply }\end{array}$ & -4 & -11 \\
\hline F6 & $\begin{array}{l}\$ 29.99 \text { for a } 1 \text {-month } \\
\text { supply }\end{array}$ & -6 & -13 \\
\hline
\end{tabular}

Table 10: Strongest and weakest performing elements for Vitamin D3, based on segmenting respondents according to the emotions which link with their ratings.

This chapter deals with a different application of Mind Genomics, the sales application, where the objective is to present the respondent with a modest amount of information about the product, rather than presenting the respondent with compelling information. In many selling situations there are no truly compelling bits of information that resonate with the mind of the respondent. Rather, there is the ordinary which must be romanced, along with the name of the product, and the price. In typical Mind Genomics studies the ordinary, the name, and the price are those elements which score poorly.

This chapter suggests that when the entire corpus of information comprises elements of a sales nature, without any strongly compelling elements, the respondents down-rate the vignettes. It's not that the respondents choose the best of the vignettes to uprate. Rather, all of the vignettes, typically perform poorly, with the additive constant being low, and the elements generating low impact values on the Interest Model. 
Segmenting respondents in typical Mind Genomics studies ends up with two or more groups, responding strongly to different elements. The segmentation often reveals groups whose response to the elements are polar opposites, and in effect groups who cancel each other, so that the average impact value is near 0 . When we deal with selling messages, again typically without compelling information, we find that there are segments suggesting different points of view, but the elements do not suddenly achieve high impact levels. Rather, the elements remain poor performers.

Segmenting respondents by the pattern of feelings/emotions linking with the elements may be more fruitful for selling messages. The impacts of the elements tend to be higher, although not dramatically higher. Segmenting respondents by feelings/emotions may hold greater promise when it comes to working with messages which are more prosaic, ordinary, and simply designed to sell rather than convey important news.

\section{Bibliography}

1. Kenny AM., et al. "Effects of vitamin D supplementation on strength, physical function, and health perception in older, community-dwelling men". Journal of the American Geriatrics Society 51 (2003): 1762-1767.

2. Lansdowne AT and Provost SC. "Vitamin D3 enhances mood in healthy subjects during winter". Psychopharmacology 135 (1998): 319-323.

3. Rosen CJ. "Vitamin D insufficiency". New England Journal of Medicine 364 (2011): 248-254.

4. Wilkins, C.H., et al. "Vitamin D deficiency is associated with low mood and worse cognitive performance in older adults". The American Journal of Geriatric Psychiatry 14 (2006): 10321040.

5. Wolpowitz D and Gilchrest BA. "The vitamin D questions: how much do you need and how should you get it?" Journal of the American Academy of Dermatology 54 (2006): 301-317.

6. Moskowitz $\mathrm{H}$ and Moskowitz D. "Understanding the mind of the consumer with regard to OTC products: Sleep-Aids". Chronicles of Pharmaceutical Sciences 2 (2018): 627-651.

7. Moskowitz JR., et al. "Development applications of emergent scenarios, synergies, and suppressions uncovered within conjoint analyses". Paper presented at the Seventh Sensometrics Conference, Davis, CA, August, (2004).

8. Moskowitz HR and Gofman A. "A System and Method for Performing Conjoint Analysis". Patent Pending. Based on U.S. Provisional Application (2004).

\section{Volume 3 Issue 8 August 2019}

\section{(C) All rights are reserved by Daniel Moskowitz and Howard Moskowitz.}

